\title{
Estudio exploratorio sobre estrategias de afrontamiento del estrés generado por la COVID-19 en estudiantes de la BENEPJPL
}

Susana Martínez Martínez y María Guadalupe Santos Rebollar

Resumen

$\mathrm{A}$ partir del confinamiento por la COVID-19, las demandas académicas, psicológicas y tecnológicas han llevado al estudiante a experimentar diversas problemáticas. Por lo anterior, este artículo es resultado de una investigación que se enfocó en las estrategias de afrontamiento para el manejo del estrés generado en estudiantes de las licenciaturas en Educación Preescolar y Primaria de la "Benemérita Escuela Normal Estatal, Profesor Jesús Prado Luna (BENEPJPL)". Por lo anterior, se realizó un estudio para identificar las estrategias que los estudiantes de la BENEPJPL utilizan a fin de resistir el estrés generado por la CoviD-19, por ello, el estudio se inscribió en el paradigma cuantitativo. El conocimiento acerca de las estrategias de afrontamiento que utilizan los normalistas puede considerarse un predictor de futuras conductas; éstos deben ser incentivados a utilizar los recursos que tengan disponibles para hacer de su trayectoria estudiantil una experiencia positiva y exitosa.

Palabras clave: contingencia, estrés académico, estrategias de afrontamiento.

\section{Introducción}

La declaratoria de la pandemia por la Covid-19 originó el confinamiento y la ruptura de la cotidianidad de la vida académica, por lo que, ante el cierre de las instituciones educativas, el proceso de enseñanza-aprendizaje cambió a modalidad virtual. En investigaciones realizadas en China y Japón (Kang et al., 2020; Shigemura et al., 2020, en Téllez, 2020) con personal de salud, se concluyó que la pandemia afecta física, mental y emocionalmente debido a la incertidumbre de contraer el virus. Con la finalidad de ahondar más sobre la temática a investigar, se establecieron dos descriptores: salud mental durante la pandemia y estrés académico en estudiantes universitarios durante la pandemia. Los criterios de selección fueron: fecha de publicación de los artículos (2020), estudios nacionales e internacionales en educación superior y que formaran parte de revistas arbitradas. De los seis estudios encontrados, dos son nacionales y cuatro internacionales (España, Colombia, Cuba y Puerto Rico). Respecto al descriptor de salud mental durante la pandemia, una investigación en la Universidad de Almería, España, con 343 estudiantes, afirma que la aparición de la pandemia ocasionó una serie de problemáticas en distintos ámbitos (Lozano et al., 2020). En México, Dávalos (2020) expuso la investigación: "Efectos del confinamiento social por CoviD-19 en la comunidad normalista", donde se aplicó una encuesta a 9954 alumnos de 28 estados. Se encontró que $85 \%$ padecieron estrés y agotamiento por cumplir con las actividades de aprendizaje en tiempo y forma; $75 \%$ externó frustración por la carga de trabajo excesiva y el poco o nulo aprendizaje; por último, $68 \%$ manifestó inseguridad por saber si las actividades las habían hecho correctamente.

Con base en el descriptor de estrés académico en estudiantes universitarios durante la pandemia, en un estudio realizado por González (2020) se encuestaron a 40 estudiantes de pedagogía de la Universidad Autónoma de Chiapas, México; los resultados definieron el estrés como una situación de tensión física y emocional relacionada con las demandas escolares. Por su parte, Pérez y otros 
investigadores (2020) muestran que los estudiantes de la Universidad de Ciencias Médicas de Cuba tienen dos visiones del estrés frente a la pandemia: causa o consecuencia; es decir, tanto un sistema inmunológico vulnerable y el conocimiento sobre la enfermedad pueden generar estrés. En México, se realizó un estudio cuantitativo sobre el impacto de la Covid-19 en el estrés de universitarios, donde se determinó que, por la educación virtual, aumentó el estrés debido a la carga académica, dificultad para dar seguimiento a las sesiones sincrónicas, situación económica, incremento en las responsabilidades domésticas y sensación de pérdida de la libertad (Montalvo y Montiel, 2020). En otro estudio cuantitativo sobre las demandas que experimentan estudiantes de 17 universidades en Puerto Rico durante la pandemia, se identificaron que los estresores más significativos fueron: obtener bajas calificaciones, falta de recursos económicos para adquirir tecnología o para pagar el internet, asistir a las sesiones virtuales, así como los retrasos en las actividades académicas e incomprensión de los temas (Rosario et al., 2020).

Las demandas académicas derivadas de la implementación de modelos de aprendizaje a distancia han generado estrés académico. En este sentido, se han hecho investigaciones con estudiantes de educación superior, a fin de identificar cuáles estrategias utilizan para afrontar el estrés, sin embargo, no se tiene suficiente información sobre estudiantes de Escuelas Normales. En este aspecto, el Banco Interamericano de Desarrollo (2020) sugiere conocer la realidad de los estudiantes durante la situación de la pandemia para que las instituciones tomen acciones en beneficio de ellos. En la Estrategia de Desarrollo Institucional de la Escuela Normal (EDINEN, 2020-2021) se establecen categorías de análisis para promover la calidad educativa. De la primera categoría, Gestión y Fortalecimiento Institucional, se desprende el programa de tutoría, asesoría y apoyo a estudiantes de nuevo ingreso y regulares; desde las acciones implementadas en esta categoría se pueden identificar las condiciones de estrés en las que se encuentran los estudiantes, así como las estrategias de afrontamiento para combatirlo. Por lo anterior, se realizó un estudio para identificar las estrategias que los estudiantes de la BENEPJPL utilizan para afrontar el estrés generado por la COVID-19. El objetivo específico fue identificar la existencia de diferencias significativas en las estrategias de afrontamiento al estrés de los alumnos de la BENEPJPL con relación a la licenciatura que cursan.

《El estrés que experimentan los estudiantes durante su formación académica, aunado al periodo de confinamiento, puede constituir un problema, y poner en riesgo su bienestar $\gg$

\section{Desarrollo}

El estrés que experimentan los estudiantes durante su formación académica, aunado al periodo de confinamiento, puede constituir un problema, lo que pone en riesgo su bienestar. Lazarus y Folkman (1986) definieron el estrés como un conjunto de factores que se establecen entre la valoración que hace el individuo de una situación y su capacidad para enfrentarla. El Fondo de las Naciones Unidas para la Infancia (UNICEF, 2017) lo define como un padecimiento común que obstruye al alumno durante su trayectoria. Sarubbi de Rearte y Castaldo (2013) señalan que se genera tensión cognitiva, emocional y conductual que afecta el bienestar emocional. Por lo tanto, el alumno requiere evaluar las acciones ante cierta situación de conflicto y relacionarlas con la percepción positiva o negativa para enfrentarlo. Un nivel elevado de estrés académico trastorna las respuestas del individuo, lo cual repercute de forma negativa en el rendimiento académico. Los factores que predisponen a un individuo al estrés pueden estar determinados por su personalidad, autoestima, autoconcepto y resistencia; por tanto, un factor de estrés puede ser percibido diferente por dos personas, ya que las respuestas a los estímulos estresantes son individuales y subjetivas, lo que refleja la capacidad de adaptación de cada sujeto (Domingues-Hirsch et al., 2018). Por lo anterior, los estudiantes requieren atención y apoyo de la sociedad, las familias y las instituciones en las que cursan su carrera.

Respecto a la selección idónea de una estrategia, para Sommerfield y McCrae esto depende del contexto concreto en el que se aplica, ya que lo que es adecuado en una situación, no necesariamente lo es en otra (2000). Por su parte, Berrío y Mazo (2011) afirman que "los estilos de afrontamiento son disposiciones personales estables que permiten hacer frente a las diferentes situaciones estresantes" (p. 77). Asimismo, López (2017) menciona que las estrategias pueden funcionar como un aliciente a los malestares del estrés o a prevenir su aparición. Por su parte, Calpa y otros investigadores (2019) señalan que las estrategias deben plantearse desde el autocuidado y la autogestión. Lazarus y Folkman (1986) afirman que el afrontamiento es una función adaptativa del individuo ante una situación de conflicto en la que intervienen los aspectos individuales y el ambiente social. En consonancia, López (2016) postula que "el objetivo de toda técnica de afrontamiento es que el estudiante recupere el equilibrio sistémico y alivie su malestar emocional para que pueda ser capaz de tener un rendimiento académico que vaya conforme a su potencial real" (p. 455). Cabanach y otros 
investigadores (2010, en González, 2020) mencionan que existen dos tipos de estrategias de afrontamiento: orientadas al problema o activas, representadas en la reevaluación positiva y planificación de acciones para eliminar el estrés; y orientadas a la emoción, buscando ayuda, apoyo social y emocional.

Las estrategias de afrontamiento orientadas al problema implican la capacidad de diferir pensamientos negativos y mantener un enfoque positivo frente a él. Para Cabanach y otros investigadores (2010), las principales estrategias de afrontamiento activo se podrían clasificar en tres grupos: 1) reevaluación positiva, que incluye aquellas acciones dirigidas a crear un nuevo significado de las situaciones estresantes; 2) búsqueda de apoyo social instrumental y emocional, centrada en la información, comprensión y alivio emocional por parte de otros; y 3) acciones que se fundamentan en la modificación del problema a partir de una aproximación analítica y racional a la misma. Fernández y Polo (2011) afirman que las estrategias dirigidas a solucionar problemas no sólo disminuyen los efectos, sino también las consecuencias: síntomas depresivos, fobias y malestar psicológico. Las estrategias de afrontamiento orientadas en la emoción se relacionan principalmente con enfermedades crónicas, no como la covid-19, la cual se refiere a una causa de alteración psicológica. Estas estrategias permiten evitar o alejarse del problema y resaltar los aspectos positivos, con el fin de cambiar la manera en la que se vive la situación estresante, aun en aquellos casos en los que no se pueda hacer nada para transformarla (Lazarus y Folkman,1986).

El estudio presentado en este artículo se inscribe en una investigación cuantitativa, exploratoria-descriptiva, cuyo escenario fue la BENEPJPL de Ensenada, Baja California (BC). La técnica utilizada para obtener la información necesaria para su análisis fue la encuesta. El cuestionario utilizado como herramienta para llevar a cabo la encuesta se tituló: "Formulario sobre estrategias de afrontamiento para el manejo del estrés". Para diseñar los reactivos que componen el cuestionario, se revisaron teóricamente los principales conceptos de la temática, posteriormente se realizó una tabla de congruencia especificando categorías, subcategorías y códigos; se analizaron diversos instrumentos (Alves, Cruz y Aguiar, 2006; Barraza, 2018; González, Rosario y Cruz, 2020; Instituto Mexicano del Seguro Social (IMSs), 2018; Pacheco, 2017; Rosario et al., 2020; Sandín y Chorot, 2003). El cuestionario se capturó en formulario Google; para la validación de su contenido se invitó a dos especialistas y a cuatro estudiantes de dos Normales de BC; el proceso se llevó a cabo del
22 al 29 de enero de 2021 en las modalidades presencial y virtual. A los jueces se les presentó la tabla de congruencia y el formato de validación con los criterios: claridad, coherencia, relevancia y suficiencia. Al concluir esta etapa de validación por jueceo, se elaboró la versión final, organizada en cinco secciones: 1) introducción, 2) aviso de privacidad, 3) datos personales, 4) estrés de pandemia y 5) estrategias de afrontamiento, en total 104 ítems, de los cuales únicamente 23 corresponden a los resultados de este estudio (ejemplos de ítem: 1 . Soy capaz de autorregularme si es necesario; 2. Me resulta suficiente un corto descanso para reponerme). Se utilizó una escala tipo Likert: nunca, casi nunca, algunas veces, casi siempre y siempre.

Del 2 al 8 de febrero de 2021 se aplicó virtualmente el instrumento a 313 estudiantes; la participación fue voluntaria y la información recopilada se manejó confidencialmente. Se finalizó la recepción de respuestas el 17 de febrero. Atendieron 189 alumnos (84 de la licenciatura en Educación Preescolar [LEPREE] y 105 de la licenciatura en Educación Primaria [LEPRIM]) que representan $60.38 \%$ de la matrícula.

Con las respuestas se integraron todos los resultados en una tabla de Excel, la cual se depuró; ésta se registró en el Programa SPSS (versión 25) para realizar diferentes pruebas de estadística descriptiva, como cálculo de media y desviación estándar, e inferencial, como Prueba t (ver Tabla 1). La diferencia de los resultados reside en las puntuaciones obtenidas en algunos ítems que lograron significación estadística. Cabe señalar que los resultados se consideraron a partir de los que fueron superiores a una media de 2 puntos.

Tanto en la LEPREE como en la LEPRIM se identificó que los estudiantes eligen las estrategias de afrontamiento para el manejo del estrés orientadas al problema o activas, destacando, respectivamente, las de buscar soluciones para las situaciones estresantes con pareja, familia y/o amigos, y ser capaz de autorregularse. Al recurrir a este tipo de estrategias, el estudiante desarrolla habilidades cognitivas y conductuales, ya que planea alternativas de solución para manejar los estresores; por lo tanto, las valoraciones positivas que haga acerca de sus propias competencias serán la principal evaluación que le permitirá afrontar el estrés “... sujetos cuyas creencias de autoeficacia sean elevadas, serán capaces de sostener y aplicar oportuna y eficazmente el estilo de afrontamiento empleado para alcanzar la meta propuesta" (Piergiovanni y Depaula, 2018, p. 21). Este tipo de estrategias son las más eficaces para manejar el estrés académico, ya que reduce su impacto y le permite al estudiante mantener un equilibrio físico y mental, indispensable para adaptarse a las exigencias escolares y rendir exitosamente. 
Tabla 1. Resultados de la elección de estrategias de afrontamiento por licenciatura

\begin{tabular}{|c|c|c|c|c|}
\hline \multirow{3}{*}{ Variable } & \multicolumn{2}{|c|}{ Grupos } & \multicolumn{2}{|c|}{ Resultados estadísticos } \\
\hline & $\begin{array}{l}\text { Licenciatura } \\
\text { en Educación } \\
\text { Preescolar } \\
(\mathbf{n = 8 4 )}\end{array}$ & $\begin{array}{c}\text { Licenciatura } \\
\text { en Educación } \\
\text { Primaria } \\
(\mathbf{n}=\mathbf{1 0 5})\end{array}$ & \multirow[t]{2}{*}{ Estadístico $t$} & \multirow[t]{2}{*}{$\begin{array}{l}\text { Diferencia } \\
\text { de medias }\end{array}$} \\
\hline & \multicolumn{2}{|c|}{ Media (desviación estándar) } & & \\
\hline \multicolumn{5}{|c|}{ Estrategias orientadas al problema o activas } \\
\hline Autorregulación & $2.69(0.98)$ & $2.74(0.77)$ & -0.40 & -0.052 \\
\hline Descanso corto & $2.14(1.08)$ & $2.21(1.04)$ & -0.43 & -0.067 \\
\hline Persona asertiva & $2.18(0.88)$ & $2.24(0.83)$ & -0.48 & -0.060 \\
\hline Ejercicio & $2.45(1.23)$ & $2.54(1.27)$ & -0.49 & -0.090 \\
\hline Pasatiempos & $2.46(1.23)$ & $2.47(1.23)$ & -0.01 & -0.002 \\
\hline Organización del tiempo & $2.32(1.13)$ & $1.92(1.04)$ & $2.51 *$ & 0.398 \\
\hline Buscar información & $2.45(1.10)$ & $2.11(1.06)$ & $2.14 *$ & 0.338 \\
\hline Soluciones similares & $2.49(1.13)$ & $2.28(1.05)$ & 1.34 & 0.212 \\
\hline Plan de resolución & $2.14(1.24)$ & $1.81(1.03)$ & $1.98^{*}$ & 0.333 \\
\hline Enfoque positivo & $2.39(1.14)$ & $2.26(1.03)$ & 0.86 & 0.136 \\
\hline Soluciones con otros & $2.81(0.94)$ & $2.70(1.02)$ & 0.73 & 0.105 \\
\hline No sobrepasen problemas & $2.74(1.14)$ & $2.50(1.00)$ & 1.56 & 0.243 \\
\hline \multicolumn{5}{|l|}{ Estrategias orientadas a la emoción } \\
\hline Apoyo por gente & $3.07(1.12)$ & $2.84(1.08)$ & 1.45 & 0.233 \\
\hline Contar problemas & $3.23(1.18)$ & $2.86(1.22)$ & $2.10^{*}$ & 0.369 \\
\hline Hablar abiertamente & $2.74(1.34)$ & $2.54(1.31)$ & 1.01 & 0.195 \\
\hline Preguntar manejo de emociones & $1.95(1.35)$ & $1.69(1.35)$ & 1.35 & 0.267 \\
\hline Escribir manejo de emociones & $1.52(1.45)$ & $1.35(1.39)$ & 0.83 & 0.171 \\
\hline Especialista & $0.67(1.20)$ & $0.98(1.49)$ & -1.62 & -0.314 \\
\hline Espacios tranquilos & $2.69(1.26)$ & $2.43(1.15)$ & 1.49 & 0.262 \\
\hline Meditación & $0.73(1.14)$ & $0.83(1.12)$ & -0.62 & -0.102 \\
\hline Oración & $2.38(1.39)$ & $2.10(1.49)$ & 1.41 & 0.298 \\
\hline Servicios religiosos & $1.01(1.53)$ & $1.10(1.50)$ & -0.38 & -0.083 \\
\hline Técnicas de manejo de emociones & $1.64(1.37)$ & $1.52(1.27)$ & 0.62 & 0.119 \\
\hline
\end{tabular}

Nota: Con negritas se señala el resultado más alto, * para $p<0.05$

Fuente: Elaborada por Martínez y Santos (2021) a partir de los resultados.

Fueron las estudiantes de la LEPREE quienes eligieron las estrategias de afrontamiento orientadas a la emoción, siendo la más representativa la de contar un problema personal a un familiar, amigo o pareja. En el caso de la LEPRIM, ninguna estrategia excedió la escala intermedia establecida. Quienes utilizan estas estrategias enfatizan las relaciones interpersonales de forma afectuosa. De acuerdo con Piergiovanni y Depaula (2018), estas estra- tegias permiten fortalecer el autoconcepto y autopercepción, “...[quienes] utilizan estrategias paliativas, como es el centrarse en las emociones o el escape conductual y/o cognitivo, manifiestan un mayor malestar físico" (Carver y Scheier, 1994, en Cassaretto et al., 2003, p. 386). Las estrategias de afrontamiento orientadas a la emoción que obtuvieron los resultados estadísticos más bajos fueron: 1) acudir a recibir ayuda de un especialista, 2) hacer me- 
ditación, 3) asistir a servicios religiosos, 4) escribir las emociones que les generan estrés, 5) utilizar técnicas de manejo de las emociones para relajarse y 6) preguntar a un familiar o amigo cómo manejar la emoción que le preocupa. Las variables con diferencia significativa entre licenciaturas son: organización del tiempo, buscar información, contar problemas y plan de resolución; las tres primeras orientadas al problema y la última a la emoción, ubicándose las respuestas más altas en la LEPREE.

\section{Conclusiones}

La suspensión de clases presenciales a raíz de la pandemia generó que en la BENEPJPL se realizara el estudio sobre estrategias de afrontamiento para el manejo del estrés, y con ello atender el bienestar emocional de los estudiantes. Las estrategias de afrontamiento pueden establecerse cuando se ha analizado la situación estresante, además de que se han planteado los objetivos y evaluado los recursos. Se destaca como conclusión principal de esta investigación que los estudiantes de la BENEPJPL recurren en mayor medida a las estrategias de afrontamiento orientadas a la emo- ción; ya que éstas tienen como objetivo reducir, regular o manejar el malestar emocional provocado por la situación que le genera estrés. Estas estrategias no resuelven en sí mismas una situación, pero originan bienestar. Los estudiantes son sujetos activos que perciben selectivamente las situaciones problemáticas en cómo reflejan sus historias, metas y las normas sociales; asimismo, requieren escoger las estrategias para librar obstáculos (Riego, 2017). El carácter de autoinforme del instrumento aplicado ofreció a los participantes la posibilidad de valorar su nivel de estrés y las estrategias que utilizan. Al presentar los resultados a la comunidad estudiantil señalaron la importancia de éstos, ya que percibieron que se estaba considerando su estatus emocional durante este periodo de confinamiento. Este estudio permitirá la intervención oportuna en acciones enfocadas en el bienestar en los estudiantes, ya que se están desarrollando programas institucionales desde las diferentes áreas y proyectos de investigación de los cuerpos académicos que evaluarán, darán seguimiento y favorecerán el entrenamiento en estrategias de afrontamiento y manejo del estrés, así como el desarrollo de sanas trayectorias estudiantiles que atiendan aspectos de vulnerabilidad provocados por los efectos de la pandemia.

\section{Fuentes de consulta}

Alves, J., Cruz, A. y Aguiar, Z. (2006). Adaptación para la lengua portuguesa de la Depression, Anxiety and Stress Scale (DASS). Rev. Latino-Am. Enfermagem 14(6), 1-9. Recuperado de https://bit.ly/3jgyfCM

Banco Interamericano de Desarrollo. (2020). La educación en tiempos del coronavirus: Los sistemas educativos de América Latina y el Caribe ante CovID-19. División de Educación. Recuperado de http://dx.doi.org/10.18235/0002337

Barraza, A., (2018). Inventario sisco sv-21. Inventario sistémico cognoscitivista para el estudio del estrés académico. Segunda versión de 21 ítems. ECORFAn, México. Recuperado de https://bit.ly/3aYzbY1

Berrío, N. y Mazo, R. (2011). Estrés Académico. Revista de Psicología de la Universidad de Antioquía, 3(2), 55-82. Recuperado de https://bit.ly/3CcnB7m

Calpa, A., Santacruz, G., Álvarez, M., Zambrano, C., Hernández, E. y Mantabanchoy, S. (2019). Promoción de estilos de vida saludables: estrategias y escenarios. Redalyc, 24(2), 1-21. https://www.redalyc.org/articulo. oa? id=309162719011

Cassaretto, M., Chau, C., Oblitas, H. y Valdez, N. (2003). Estrés y afrontamiento en estudiantes de Psicología. Pontificia Universidad Católica del Perú. Revista de Psicología de la PUCP, 21(2), 364-392. https://doi.org/10.18800/ psico. 200302.006

Dávalos, A. (2020). Efectos de confinamiento social por Covid-19 en la comunidad estudiantil normalista. Recuperado de https://bit.ly/3CcnlFq

Domingues-Hirsch, C., Devos-Barlem, E. L., De Almeida, L. K., Tomaschewski-Barlem, J. G., Lerch-Lunardi, V., \& Marcelino-Ramos, A. (2018). Stress triggers in the educational environment from the perspective of nursing students. Texto \& Contexto Enfermagem, 27(1).

Fernández, C. y Polo, M. (2011). Afrontamiento, estrés y bienestar psicológico en estudiantes de educación social de nuevo ingreso. EduPsykhé: Revista de Psicología y Psicopedagogía, 10(2), 177-192. Recuperado de https://bit. ly/3pfWfKa 
González, L. (2020). Estrés académico en estudiantes universitarios asociados a la pandemia por coviD-19. Espacio $i+d$, Innovación más Desarrollo, 9(25), 148-179. Recuperado de https://doi.org/10.31644/IMASD.25.2020.a10

González, J., Rosario, A. y Cruz, A. (2020). Escala de Ansiedad por Coronavirus: Un Nuevo Instrumento para Medir Síntomas de Ansiedad Asociados al Covid-19. Interacciones, 6(3), 1-8. Recuperado de https://doi. org/10.24016/2020.v6n3.163

Instituto Mexicano del Seguro Social. (2018). Test de estrés laboral. IMSs. Recuperado de https://bit.ly/3E5f5Id Lazarus, R. y Folkman, S. (1986). Estrés y procesos cognitivos. Ediciones Martínez Roca.

López, A. (2016). Estrés académico en maestros en formación de la IByCENECH: Un estudio comparativo entre dos licenciaturas. RECIE. Revista Electrónica Cientifica de Investigación Educativa, 3(1), 453-461. Recuperado de https:// bit.ly/31YxMad

López, A. (20-24 de noviembre de 2017). Estrés académico en estudiantes normalistas del estado de Chihuahua. [Ponencia]. XIV Congreso Nacional de Investigación Educativa, San Luis Potosí, México. Recuperado de https://bit. ly/3aSE8By

Lozano, A., Fernández, J., Figueredo, V. y Martínez, A. (2020). Impactos del confinamiento por el covid-19 entre universitarios: Satisfacción Vital, Resiliencia y Capital Social Online, International Journal of Sociology of Education, Special Issue: CovID-19 Crisis and Socioeducative Inequalities and Strategies to Overcome them, 9(1), 79-104. Recuperado de http://doi.org/10.17583/rise.2020.5925

Montalvo, N. y Montiel, A. (2020). Impacto del Covid-19 en el estrés de universitarios. AvaCient, 4(2), 79-85. Recuperado de https://bit.ly/30OJj3T

Pacheco, J. (2017). Estrés académico de los estudiantes de enfermería en una universidad privada de Puerto Rico y su asociación con el rendimiento académico. Universidad de Málaga. EDITA: Publicaciones y Divulgación Científica. Universidad de Málaga. Recuperado de https://riuma.uma.es/xmlui/handle/10630/15903

Pérez, M., Gómez, J., Tamayo, O., Iparraguirre, A. y Besteiro, E. (2020). Alteraciones psicológicas en estudiantes de medicina durante la pesquisa activa de la COVID-19. MEDISAN, 24(4), 537-548. Recuperado de http://dx.doi. org/10.17583/rise.2020.5925

Piergiovanni, L. y Depaula, P. (2018). Estudio descriptivo de la autoeficacia y las estrategias de afrontamiento al estrés en estudiantes universitarios argentinos. Revista mexicana de investigación educativa, 23(77), 413-432. https:// www.comie.org.mx/revista/v2018/rmie/index.php/nrmie/article/view/1155

Riego, M. (20-24 de noviembre de 2017). Habitus bourdiano y estrategias de afrontamiento en estudiantes de ingeniería. [Ponencia]. XIV Congreso Nacional de Investigación Educativa, San Luis Potosí, México. Recuperado de https://bit.ly/3vBW4Kf

Rosario, A., González, J., Cruz, A., y Rodríguez, L. (2020). Demandas tecnológicas, académicas y psicológicas en estudiantes universitarios durante la pandemia por Covid-19. Revista Caribeña de Psicología, 4(2), 176-185. Recuperado de https://doi.org/10.37226/rcp.v4i2.4915

Sandín, B. y Chorot, P. (2003). Cuestionario de afrontamiento del estrés (CAE): desarrollo y validación preliminar. Revista de Psicopatología y Psicología Clínica 8(1), 39-54. Recuperado de http://revistas.uned.es/index.php/ RPPC/article/view/3941

Sarubbi de Rearte, E. y Castaldo, I. (2013). Factores causales del estrés en los estudiantes universitarios. v Congreso Internacional de Investigación y Práctica profesional en Psicología. XX Jornadas de Investigación Nuevo encuentro de investigadores en Psicología MERCASUR. Recuperado de https://www.aacademica.org/000-054/466

Secretaría de Educación Pública. (2020). Guía Metodológica de la Estrategia de Desarrollo Institucional de la Escuela Normal (EDINEN) 2020-2021.

Sommerfield, M., y McCrae, R. (2000). Stress and coping research. Methodological challenges, theoretical advances, and clinical applications. American Psychologist, 55 (6), 620-625.

Téllez, J. (2020). Pandemia: estrés prolongado y sobrecarga inmunológica. Yo te cuido, tú me cuidas. Recuperado de https://bit.ly/39My7WJ

UNICEF. (2016). Estado Mundial de la infancia 2017. Niños en un mundo digital. UNICEF. https://www.unicef.org/me$\mathrm{dia} / 48611 /$ file 


\section{ESTRATECLAS DE AFRONTAMENTO DEL}

\section{Citiés}

Las estrategias orientadas al problema o activas son las más eficaces para manejar el estrés académico, ya que reduce su impacto y le permite al estudiante mantener un equilibrio físico y mental, indispensable para adaptarse a las exigencias escolares y rendir exitosamente.
Las estrategias orientadas a la emoción tienen como objetivo reducir, regular o manejar el malestar emocional provocado por la situación que le genera estrés. Estas estrategias no resuelven en sí mismas una situación, pero originan bienestar.

\section{EXPRESAR LOS} PROBLEMAS

\section{TIEMPO}

(1)

Al recurrir a este tipo de estrategias, el estudiante cognitivas y conductuales ya que planea alternativas de solución para manejar los estresores.

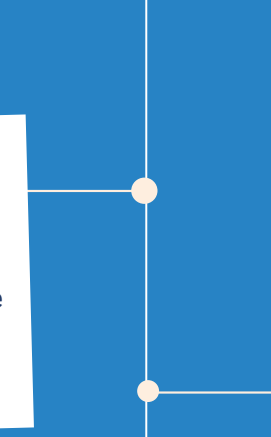
desarrolla habilidades

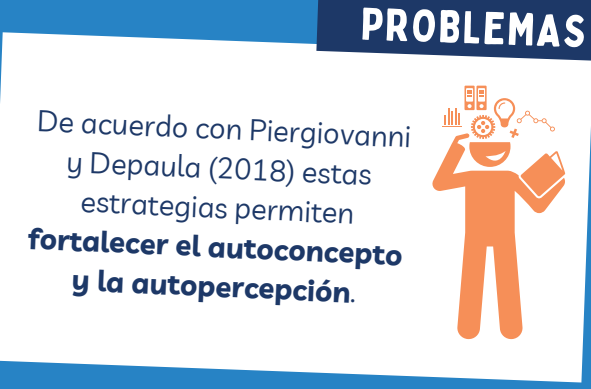

APOYARSE

\section{BUSCAR} INFORMACIÓN

Por lo anterior, las valoraciones positivas que haga acerca de sus propias competencias serán la principal evaluación que le permitira afrontar el estrés.

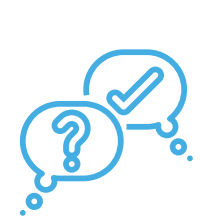
. EN LA GENTE

Los estudiantes de la BENEPJPL recurren en mayor medida a las estrategias de afrontamiento orientadas a la emoción.

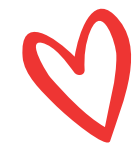

Quienes utilizan estas estrategias enfatizan las relaciones interpersonales de forma afectuosa. 